\title{
un modèle élastoplastique anisotrope avec écrouissage pour les argiles molles naturelles: Mélanie
}

\author{
par \\ A. Mouratidis \\ J.P. Magnan \\ Laboratoire central des Ponts et Chaussées, Paris
}

\section{Introduction}

Du fait de sa complexité, le comportement des sols fins a fait l'objet de programmes de recherche variés, qu'il s'agisse de tentatives purement théoriques, d'études menées en laboratoire, d'expériences en vraie grandeur ou encore d'approches semi-empiriques combinant de manière rationnelle théorie et réalité.

Durant les années soixante, le Groupe de Mécanique des Sols de I'Université de Cambridge a transformé en modèles mathématiques certaines des idées sur les lois "contraintes-déformations" du sol développées par différents chercheurs:

- le concept de surface d'état limite.

- la notion d'état critique,

- le concept d'état stable,

- le principe du travail maximal.

Fondés sur ces principes, les modèles de Cambridge, dont la caractéristique principale est l'introduction de l'a élastoplasticité avec écrouissage" comme loi de comportement du sol, proposent un nouveau mode d'analyse des problèmes concernant les argiles molles, largement suivi depuis par les mécaniciens des sols.

Au L.C.P.C., c'est le modèle Cam-Clay modifié, introduit dans le programme de calcul par éléments finis Rosalie, qui a servi en premier lieu d'outil pour les études rhéologiques sur les sols compressibles. Élaboré à partir de principes énergétiques relativement simples, ce modèle est capable de reproduire assez fidèlement le comportement des argiles remaniées isotropes et présente, en outre, l'avantage d'être facilement utilisable dans la mesure où sa modélisation ne nécessite que des paramètres de compressibilité déduits d'essais œdométriques et triaxiaux classiques. Cette expérience, qui a donné des résultats satisfaisants dans le cas du calcul du remblai B de Cubzac-les-Ponts (Dang et Magnan, 1977; Magnan et Belkeziz, 1982) a néanmoins laissè en suspens deux points d'interrogation :

- la surface d'état limite du modèle Cam-Clay modifié, conçue pour les argiles isotropes normalement consolidées, est-elle convenable pour les sols anisotropes?

- la loi de normalité adoptée dans ce modèle comme loi d'écoulement est-elie valable pour les argiles naturelles?

Par ailleurs, la présentation par Tavenas et Leroueil (1977) des premiers résultats des expériences sur les argiles de la vallée du Saint-Laurent a mis en évidence certaines particularités du comportement mécanique des sols anisotropes qui les différencient sensiblement de l'argile isotrope du modèle Cam-Clay modifié.

Dans la perspective de l'élaboration d'un modèle pour les sols compressibles, le L.C.P.C. a alors entrepris des travaux de recherche afin d'établir, à partir d'une base expérimentale, un outil mathématique capable de décrire les lois rhéologiques des argiles molles naturelles françaises. Ces travaux de recherche, entamés en 1977, ont comporté deux phases d'études en laboratoire :

- dans une première phase, les expériences ont principalement porté sur le comportement élastoplastique du sol, la définition de la surface d'état limite et la loi d'écoulement plastique (Shahanguian, 1980; Magnan et al., 1982c);

- dans la seconde phase, les essais ont été orientés sur la détermination des caractéristiques élastiques du sol et, en particulier, sur l'évaluation des cinq paramètres d'orthotropie de révolution qui caractérisent les argiles naturelles à l'état surconsolidé : $E_{n}, E_{v}, v_{n h}, v_{v h}, G_{v h}$ (Piyal et Magnan, 1984).

BASE EXPÉRIMENTALE DU MODĖLE : SURFACE D'ÉTAT LIMITE ET LOI D'ÉCOULEMENT

Les études expérimentales qui ont conduit à la constitution du modèle Mélanie (Modèle ÉLastoplasti- 
que ANIsotrope avec Écrouissage) ont débuté à l'Université Laval de Québec sous la direction de F. Tavenas, dans le cadre des travaux de thèse de S. Leroueil (1977). Les résutats obtenus à I'Université Laval (Tavenas et Leroueil, 1977) étaient particulièrement intéressants et il a paru nécessaire de vérifier leur validité sur certaines argiles molles françaises.

En 1977. Shahanguian a entrepris au L.C.P. C, une étude rhéologique en laboratoire sur l'argile molle du site expérimental de Cubzac-les-Ponts. Cette étude a été menée avec deux objectifs

- l'évaluation des paramètres de compressibilité du sol à partir d'essais œdométriques ainsi que la recherche d'une éventuelle influence du mode opératoire sur les valeurs des différents paramètres:

- l'analyse du comportement élastoplastique du sol et la définition de la surface d'état limite à l'aide d'essais triaxiaux.

La totalité des expériences ont été réalisées sur des éprouvettes prélevées dans le sol de fondation des remblais $C$ et $D$ de Cubzac-les-Ponts. Pour assurer la représentativité de toute l'analyse, un soin particulier a été pris pour le prélèvement du sol, de sorte que les échantillons appartenant à une même couche présentent une homogénéité de caractéristiques géotechniques et se trouvent tous au même état initial $\left(e_{0}, \sigma_{\text {vo }}^{\prime}\right)$.

La surface d'état limite du sol de la couche étudiée peut être déterminée à condition de connaître, d'une part, les paramètres de compressibilitê et, notamment. les pentes $\lambda$ et $x$ de la courbe vierge isotrope et des courbes réversibles isotropes respectivement et d'autre part. la courbe limite de l'ètat initial déduite des essais triaxiaux (Fig. 1).

Cette courbe d'état limite constitue un lieu géométrique des points du plan $(s, t)$ sur lesquels s'effectue l'amorce de plastification du sol et a été obtenue, dans le cas des expèriences du L.C.P.C., par différents types d'essais triaxiaux: essais de consolidation, de cisaillement, essais $K_{0}$. essais de striction.

Les résultats des essais triaxiaux réalisés sur deux ensembles d'échantillons, prélevés à deux profondeurs différentes dans le sol de fondation du remblai C $(4,5 \mathrm{~m}$ et $5,5 \mathrm{~m})$, ont été analysés dans cette optique. Cette analyse, conduite en termes de contraintes effectives, comprend les étapes suivantes

- Pour chaque palier de chargement, les contraintes effectives moyenne $s=\left(\sigma_{1}^{\prime}+\sigma_{3}^{\prime}\right) / 2$ et déviatorique $\mathrm{t}=\left(\sigma_{1}^{\prime}-\sigma_{3}^{\prime}\right) / 2$ sont calculées, les axes $\mathrm{Ox}, \mathrm{Oy}, \mathrm{Oz}$ se confondant dans ce cas avec les axes des contraintes principales $\sigma_{1}^{\prime}, \sigma_{2}^{\prime}, \sigma_{3}^{\prime}$, et $\sigma_{2}^{\prime}$ étant égal à $\sigma_{3}^{\prime}$ pour la totalité des essais triaxiaux.

- A partir de la déformation volumique $\Delta \mathrm{V} / \mathrm{V}$ et de la déformation axiale $\Delta \mathrm{h} / \mathrm{h}$ les déformations $\varepsilon_{1}$ et $\varepsilon_{2}$ sont évaluées $\left(\varepsilon_{2}=\varepsilon_{3}\right)$, ainsi que les quantités. $d v=\varepsilon_{1}+\varepsilon_{2}$ et $d \gamma=\varepsilon_{1}-\varepsilon_{2}$.

- Ces valeurs sont ensuite reportées pour tous les paliers de chargement sur des diagrammes $(s, d v)$ et $(s, d \gamma)$ d'où, par linéarisation appropriée des deux branches des courbes " contraintesdéformations". les points de passage à l'état plastique sont définis (Fig. 2). Les incréments de déformation plastique s'obtiennent en retranchant la déformation élastique de la déformation totale à l'état plastique suivant le graphique de la figure 2. Les incréments $d v^{p}$ et $d \gamma^{p}$ ainsi déduits

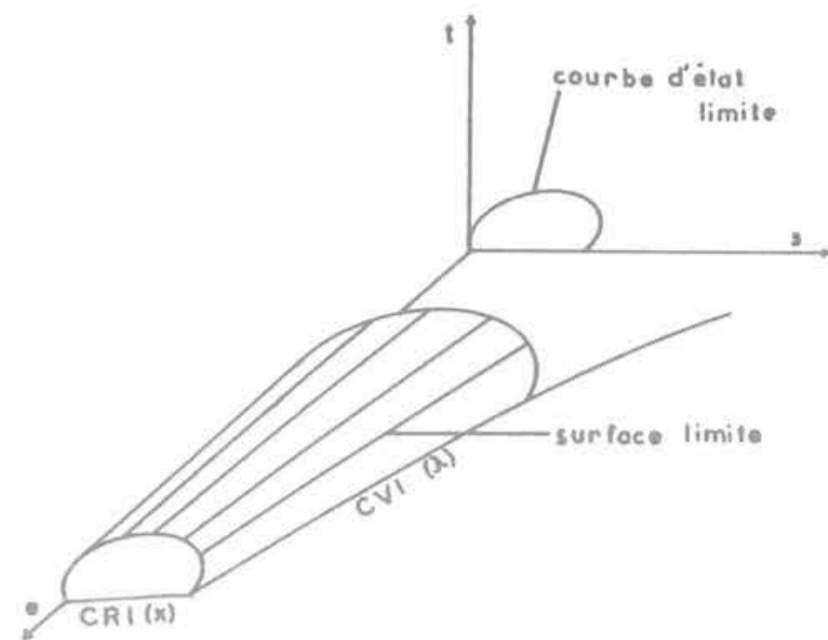

Fig. 1 Définition de la surface d'état limite
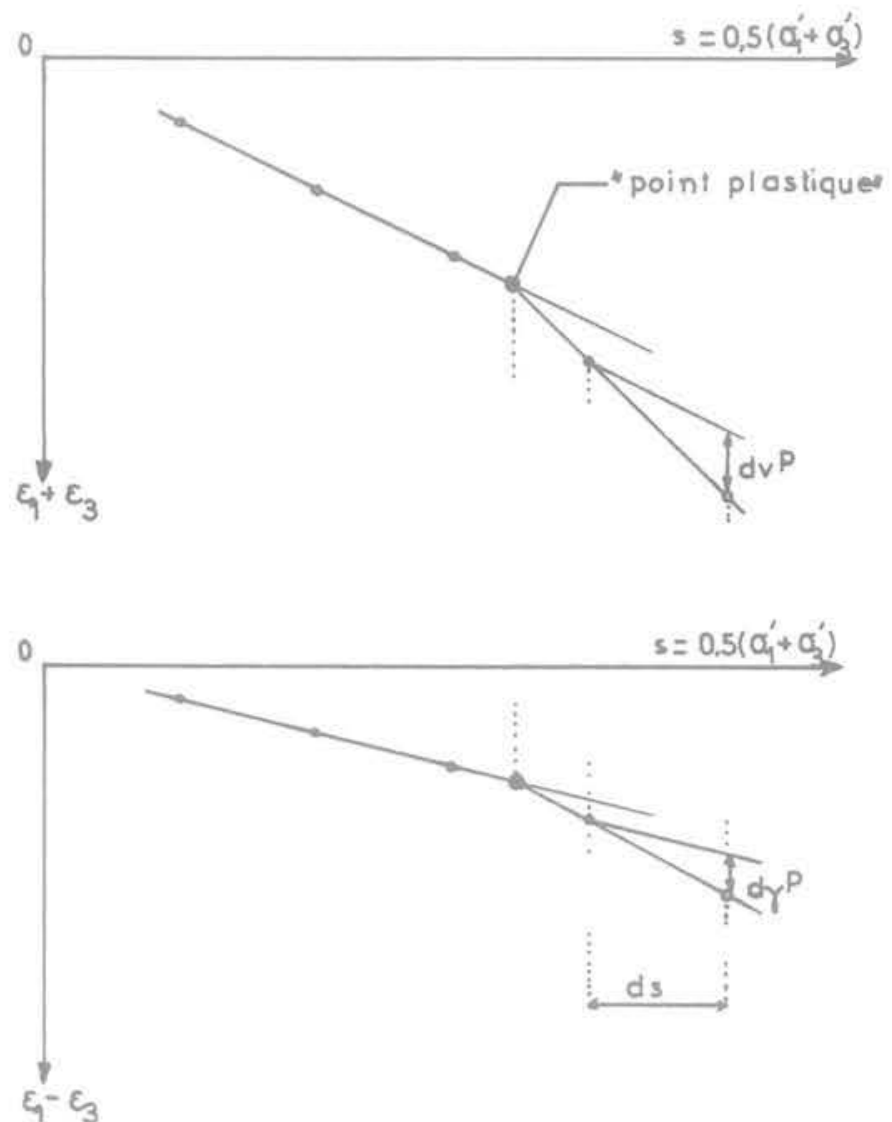

Fig. 2 Détermination des points "plastiques» et des in. créments de déformation plastique

sont respectivement les composantes moyenne et déviatorique de la déformation plastique $\mathrm{d}^{p}$, à condition, évidemment, qu'ils soient calculés pour le même incrément de contrainte ds.

- Ce travail doit être effectué pour toutes les éprouvettes appartenant au même ensemble et ainsi, finalement, chaque essai définit un point "plastique $n$, caractérisé par les valeurs $s, t, d v^{p}$, d $\gamma^{p}$

- Ces points "plastiques", reportés sur un diagramme $(s, t)$ unique pour chaque ensemble d'éprouvettes, forment la courbe d'état limite recherchée. Sur ces mêmes points, les valeurs correspondantes de $d^{D}$ et $d \gamma^{D}$, reportées suivant les axes Os et Ot respectivement, définissent les inclinaisons des vecteurs d'écoulement plastique. 


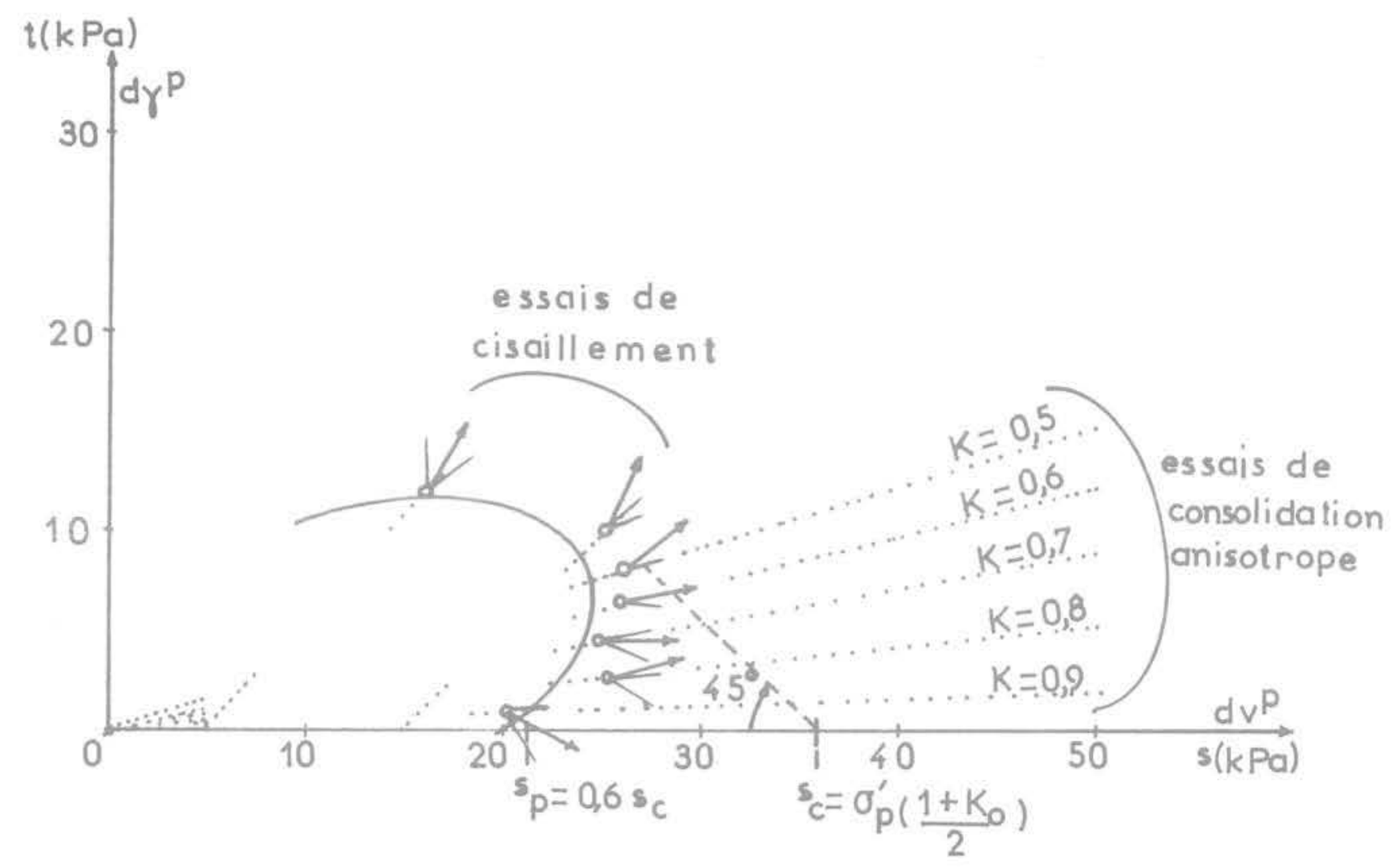

Fig. 3 Courbe d'état limite et vecteurs de déformation plastique (Remblai $C, z=4,5 \mathrm{~m}$ )

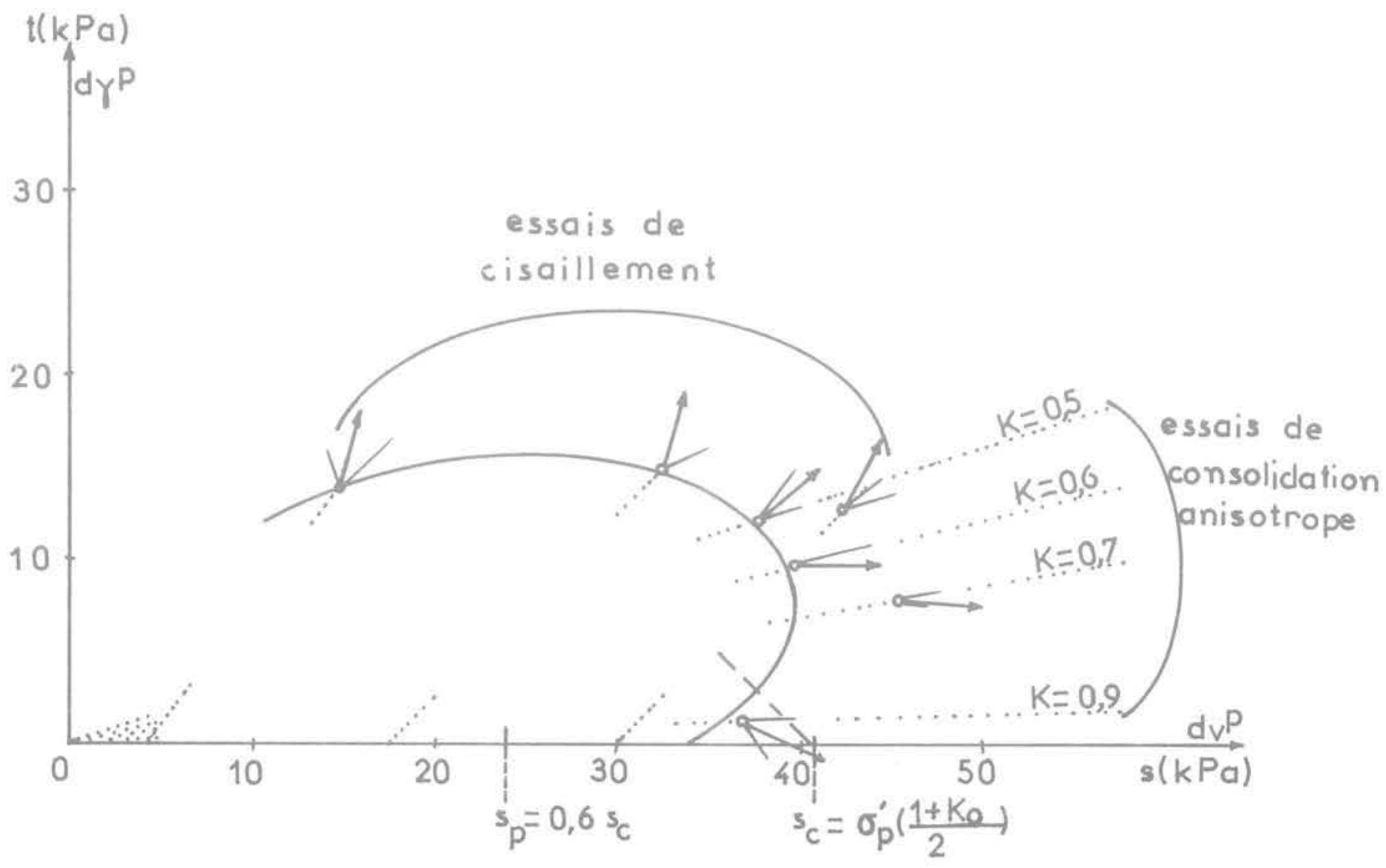

Fig. 4 Courbe d'état limite et vecteurs de déformation plastique (Remblai $C, z=5,5 \mathrm{~m}$ ) 
Les figures 3 et 4 montrent les courbes d'état limite déterminées de cette façon et les vecteurs de déformation plastique, pour les deux profondeurs du remblai C.

On peut constater que les principales hypothèses du modèle Ylight de Tavenas et Leroueil sont bien vérifiées par les résultats de ces expériences et en particulier :

- On peut admettre sans trop d'erreur que les courbes d'état limite sont de forme elliptique dans les deux cas. II est clair que les ellipses ne sont pas centrées sur l'axe des contraintes isotropes, comme l'admet le modèle Cam-Clay modifié, mais sont nettement inclinées par rapport à cet axe. On peut, toutefois, supposer qu'elles passent par l'origine des coordonnées. La première $(z=4,5 \mathrm{~m})$ semble centrée sur l'axe $K=0,6$ tandis que la seconde $(z=5,5 \mathrm{~m})$ est centrée sur l'axe $K=0,5$. Les essais $\mathrm{K}_{0}$ sur le sol de Cubzac ont montré que la valeur de $K_{0}$ est pratiquement égale à 0,5 et, par conséquent, l'hypothèse que la courbe d'ètat limite est une ellipse dont le grand axe est situé sur l'axe d'anisotropie $\mathrm{K}_{\mathrm{o}}$ semble bien confirmée.

- En ce qui concerne les dimensions de l'ellipse. Ylight suppose que celle-ci passe par deux points distincts : a) le point d'intersection de l'axe $\mathrm{K}_{\mathrm{o}}$ avec la droite qui passe par le point $\mathrm{s}_{\mathrm{c}}=\sigma_{p}^{\prime}\left(\frac{1+\mathrm{K}_{\mathrm{o}}}{2}\right)$ sur l'axe des contraintes isotropes et est inclinée de $45^{\circ}$ par rapport à cet axe et b) le point "plastique" qui correspond à l'essai isotrope défini par la relation expérimentale $s_{p}=0,6 s_{c}$. Ces observations expérimentales sont fort bien vérifiées sur la seconde courbe d'état limite où la concordance est quasi parfaite et moins bien sur la première où l'étendue du domaine élastique est plus grande que celle que l'on peut déduire de la valeur de $\sigma_{\mathrm{p}}^{\prime}$. Pour le modèle Mélanie, l'expression mathématique du critère de plasticité a été établie en supposant que l'ellipse passe par trois points: les deux points ci-dessus et l'origine des coordonnées.

- La loi d'écoulement, décrite par les vecteurs de déformation plastique, ne semble pas correspondre à la loi de normalité. La plupart des vecteurs sont situés entre la normale à la courbe au point "plastique" et la droite quí passe par le point et l'origine des coordonnées, ce qui, dans la modélisation, a été traduit par la "règle de la bissectrice " selon laquelle le vecteur $d \varepsilon^{p}$ est situé sur la bissectrice de l'angle formé par la normale et le rayon.

Ces constatations sur la forme, les dimensions et l'orientation de la courbe d'état limite, d'une part, et le caractère de la loi d'écoulement plastique, d'autre part, sont à l'origine du modèle Mélanie.

\section{LES ÉQUATIONS DU MODÈLE MÉLANIE}

La formulation mathématique du modèle est fondée sur les résultats expérimentaux décrits dans le paragraphe précédent et développés en termes d'équations suivant l'approche adoptée pour les modèles de Cambridge. La surface d'état limite dans l'espace $(\mathrm{s}, \mathrm{t}, \mathrm{e})$ est définie d'une part par la courbe d'état limite dans l'espace des contraintes $(s, t)$ et d'autre part par la courbe de compressibilité isotrope sur le diagramme classique (e, In s) (Fig. 5).

L'équation de l'ellipse de la courbe d'état limite est la suivante:
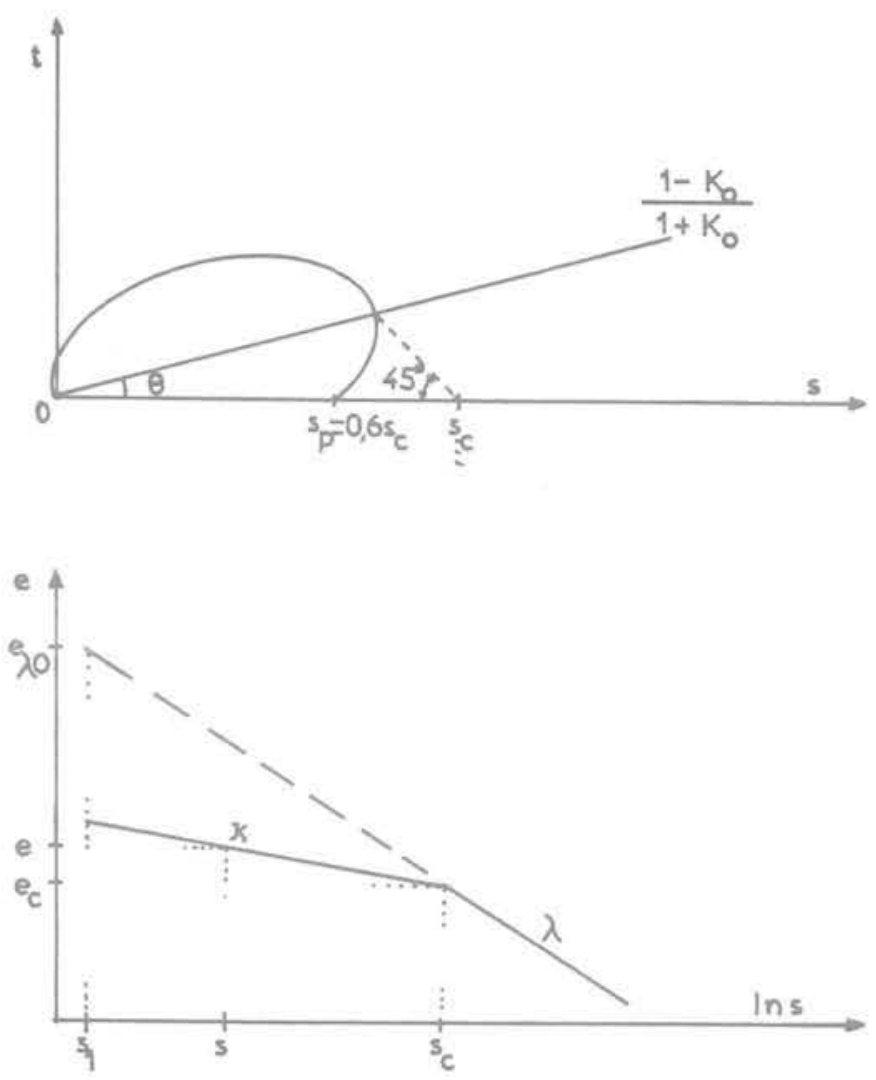

Fig. 5 Courbe d'état limite et courbe œdométrique

$A^{2}\left[s \cos \theta+t \sin \theta-\frac{s_{p}}{A C}\right]^{2}$

$$
+B^{2}[t \cos \theta-s \sin \theta]^{2}-\frac{s_{p}^{2}}{C^{2}}=0
$$

avec

$$
\begin{aligned}
A & =2(\cos \theta+\sin \theta) \\
B^{2} & =\frac{A^{2} \cos \theta}{C \sin ^{2} \theta}\left[\frac{2}{A}-C \cos \theta\right] \\
C & =0.6 \\
S_{p} & =C s_{c} .
\end{aligned}
$$

La valeur de $s_{c}$ est définie à partír de l'équation de la courbe vierge et de celle des courbes réversibles:

$$
\begin{array}{r}
e_{\lambda 0}-e_{c}=\lambda \ln \left(\frac{s_{c}}{s_{1}}\right), \\
e-e_{c}=x \ln \left(\frac{s_{c}}{s_{1}}\right),
\end{array}
$$

quí conduisent à l'expression :

$$
s_{c}=s_{1} \exp \left(\frac{e_{\lambda 0}-e}{\lambda-x}\right) \cdot\left(\frac{s}{s_{1}}\right)^{\frac{-x}{\lambda-x}}
$$

Par conséquent, l'équation de la surface d'état limite peut s'écrire :

$$
\begin{aligned}
& A^{2}\left[s \cos \theta+t \sin \theta-\frac{s_{1}}{A} \exp \left(\frac{e_{\lambda 0}-e}{\lambda-x}\right)\left(\frac{s}{s_{1}}\right)^{\frac{-x}{\lambda-x}}\right] \\
& +B^{2}[t \cos \theta-s \sin \theta]^{2}-\left[s_{1} \exp \left(\frac{e_{\lambda 0}-e}{\lambda-x}\right)\left(\frac{s}{s_{1}}\right)^{\frac{-x}{\lambda-幺}}\right]^{2}=0
\end{aligned}
$$

On peut constater que cette formulation repose, en plus des observations expérimentales déjà prises en considération, sur une hypothèse complémentaire: on admet que le rapport entre la contrainte de préconsolidation isotrope $s_{\mathrm{p}}$ et la contrainte moyenne de préconsolidation de l'œdomètre $s_{c}$ est constant et 


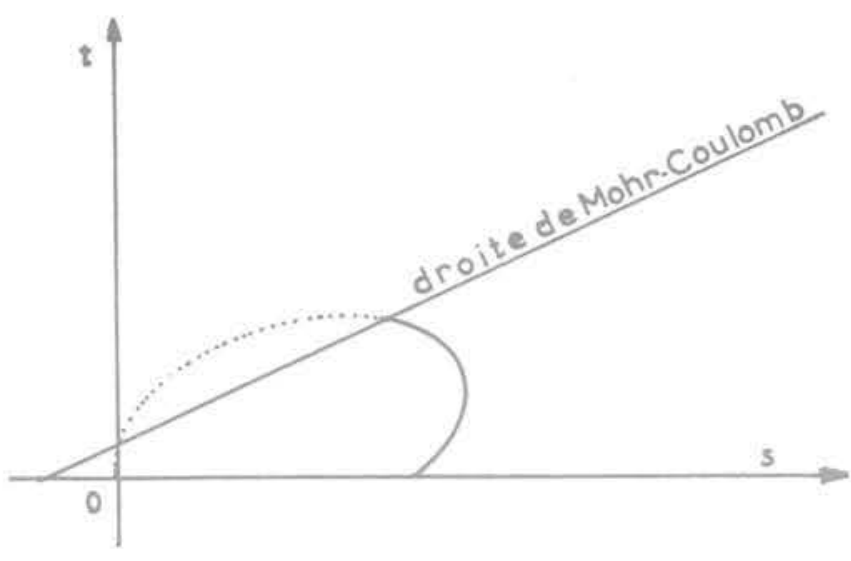

Fig. 6 Couplage avec le critère de Mohr-Coufomb

égal à 0,6 . Cette approche permet, en pratique, d'exprimer toutes les équations du modèle élastoplastique en termes de paramètres déduits uniquement de l'essai œdométrique, largement répandu et plus. courant que l'essai de consolidation isotrope.

Dans le cas des calculs élastoplastiques, le point d'état, qui caractérise à chaque instant l'état des contraintes dans le sol, peut, momentanément, se trouver dans un domaine fictif en dehors de la surface d'état limite. On se sert, alors, de contraintes de correction, qui servent à ramener le point d'état sur la surface de charge tout en respectant l'équilibre du système. L'expression de ces contraintes de correction $\Delta \sigma_{0}$ constitue une caractéristique propre de chaque modèle et un élément indispensable pour le traitement élastoplastique. Dans le cas présent, elles. sont calculées suivant la méthode des contraintes initiales sur la base d'une loi d'écoulement non associée :

$$
\mathrm{de}^{\mathrm{P}}=\mathrm{d} \lambda \cdot \mathrm{G}_{\text {. }}
$$

avec :

$d \varepsilon^{P}$; incrément de déformation plastique,

$\mathrm{d} \lambda$ : scalaire,

$\mathrm{G}_{a}$ : vecteur situé dans le cas de Mélanie sur la bissectrice du vecteur normal et du rayon.

L'expression des contraintes de correction devient:

$$
\Delta \sigma_{0}=\frac{-F(\sigma, k)}{B+F_{o}^{\top} E G_{o}} \cdot E \cdot G_{s}
$$

avec :

$F(\sigma, k)$ : fonction de charge, $k$ étant le paramètre d'écrouissage, choisi pour Mélanie égal à $S_{0}$.

E : matrice d'élasticité,

$F_{o}$ : vecteur normal à la surface $F_{\text {, }}$

$G_{1,}$ : vecteur "bissecteur",

B : terme d'écrouissage.

$\mathrm{Ce}$ critère élastoplastique avec écrouissage a été couplé avec le critère de plasticité parfaite de Mohr-Coulomb dont la fonction de charge est :

$$
\mathrm{f}=\mathrm{t}-\mathrm{s} \sin \varphi-\mathrm{c} \cos \varphi
$$

et pour lequel la loi de normalité est supposée valable (Fig. 6).

Sous sa forme actuelle, le modèle Mélanie prévoit, outre la possibilité de combiner les deux critères, l'utilisation de chacun séparément. Cette dernière alternative peut être réalisée à condition qu'une seule fonction de charge intervienne dans les calculs. l'autre s'éloignant suffisamment pour que son effet s'annule. Ceci peut être effectué en attribuant, afin de ne se servir que de l'ellipse, une très forte valeur à la cohésion c et, dans le cas contraire, une grande valeur fictive à $s_{c}$ ou $e_{\lambda_{0}}$. de sorte que l'ellipse disparaisse et seule la droite de Coulomb constitue le critère de plasticité.

A l'intérieur de la surface d'état limite le comportement du sol est supposé élastique linéaire. L'élasticité anisotrope, dans le cas présent, est une élasticité orthotrope à symétrie de révolution. Les relations contraintes-déformations sont exprimées en fonction de cinq paramètres indépendants :

$E_{n}$ : module d'Young dans le sens horizontal,

$E_{x}$ : module d'Young dans le sens vertical,

$\nu_{h t}$ : coefficient de Poisson dans la direction horizontale sous contrainte horizontale,

$v_{v h}:$ coefficient de Poisson dans la direction verticale sous contrainte horizontale,

$G_{\mathrm{vh}}$ : module de cisaillement dans le plan vertical.

Dans le cas des problèmes de déformation plane $\left(\varepsilon_{z}=\varepsilon_{x z}=\varepsilon_{y z}=0\right)$, la matrice d'élasticité $E$ permettant de calculer les contraintes $\left(\sigma_{x}, \sigma_{y}, \sigma_{x y}\right)$ à partir des déformations $\left(\varepsilon_{x}, \varepsilon_{y}, \varepsilon_{x y}\right)$ s'écrit:

$$
E=\begin{array}{ccc}
\frac{E_{h}\left(1-n v_{v}^{2}\right)}{\left(1+v_{h h}\right)\left(1-v_{h h}-2 n v_{v h}^{2}\right)} & \frac{E_{h} v_{v h}}{1-v_{h h}-2 n v_{v h}^{2}} & 0 \\
\frac{E_{h} v_{v h}}{1-v_{h h}-2 n v_{v h}^{2}} & \frac{E_{v}\left(1-v_{h n}\right)}{1-v_{h h}-2 n v_{v h}^{2}} & 0 \\
0 & 0 & G_{v h}
\end{array}
$$

avec $n=E_{h} / E_{v}$.

Les premiers calculs utilisant te modèle Mélanie, qui portaient sur les remblais expérimentaux de Cubzacles-Ponts, ont été réalisés à une époque (1981) oủ l'on ne disposait pas de valeurs expérimentales de ces paramètres d'élasticité, puisque la seconde phase des expériences entreprises par le L.C.P.C., qui comprenait les essais triaxiaux, n'avait pas encore donné de résultats. II a fallu, donc, se fier uniquement aux essais œedométriques déjà réalisés, à la littérature spécialisée, ainsi qu'à des relations entre ces caractéristiques qui résultent d'études qualitatives sur le comportement réel des remblais sur sols compressibles. Ces dernières ont dicté le choix des coefficients de Poisson $v_{\mathrm{hH}}=v_{\mathrm{yH}}=9,4$, de façon à $\mathrm{ce}$ que le rapport entre le déplacement maximal sous le pied du talus et le tassement sur l'axe de symétrie du remblai varie entre 0,15 et 0,20 . La relation $\mathrm{E}_{\mathrm{n}}=0,5 \mathrm{E}_{\mathrm{v}}$, valable pour les argiles légèrement surconsolidées, a conduit à l'évaluation des modules d'Young à partir des courbes œdométriques et $G_{v}$ a été pris égal à $0,35 E_{v}$.

Les travaux de Piyal et Magnan (1984) ont conduit à des valeurs un peu différentes de ces paramètres:

$$
\begin{aligned}
E_{\mathrm{h}} & =0,62 \mathrm{E}_{\mathrm{v}} \\
\mathrm{G}_{\mathrm{vh}} & =0,45 \mathrm{E}_{\mathrm{v}} \\
v_{\mathrm{hh}} & =0,10 \\
\nu_{\mathrm{vh}} & =0,25 .
\end{aligned}
$$

Ces résultats, obtenus au moyen d'essais triaxiaux drainés sur éprouvettes verticale, horizontale et inclinée à $45^{\circ}$, ne permettent pas de conclure que des relations analogues existent dans tous les massifs d'argiles molles. II faut indiquer d'autre part que l'interprétation des essais nécessaires à la détermination des cinq paramètres d'élasticité orthotrope de révolution est complexe et que les valeurs obtenues sont relativement dispersées. Néanmoins, l'approche adoptée est actuellement la seule possible. 
Remblai A
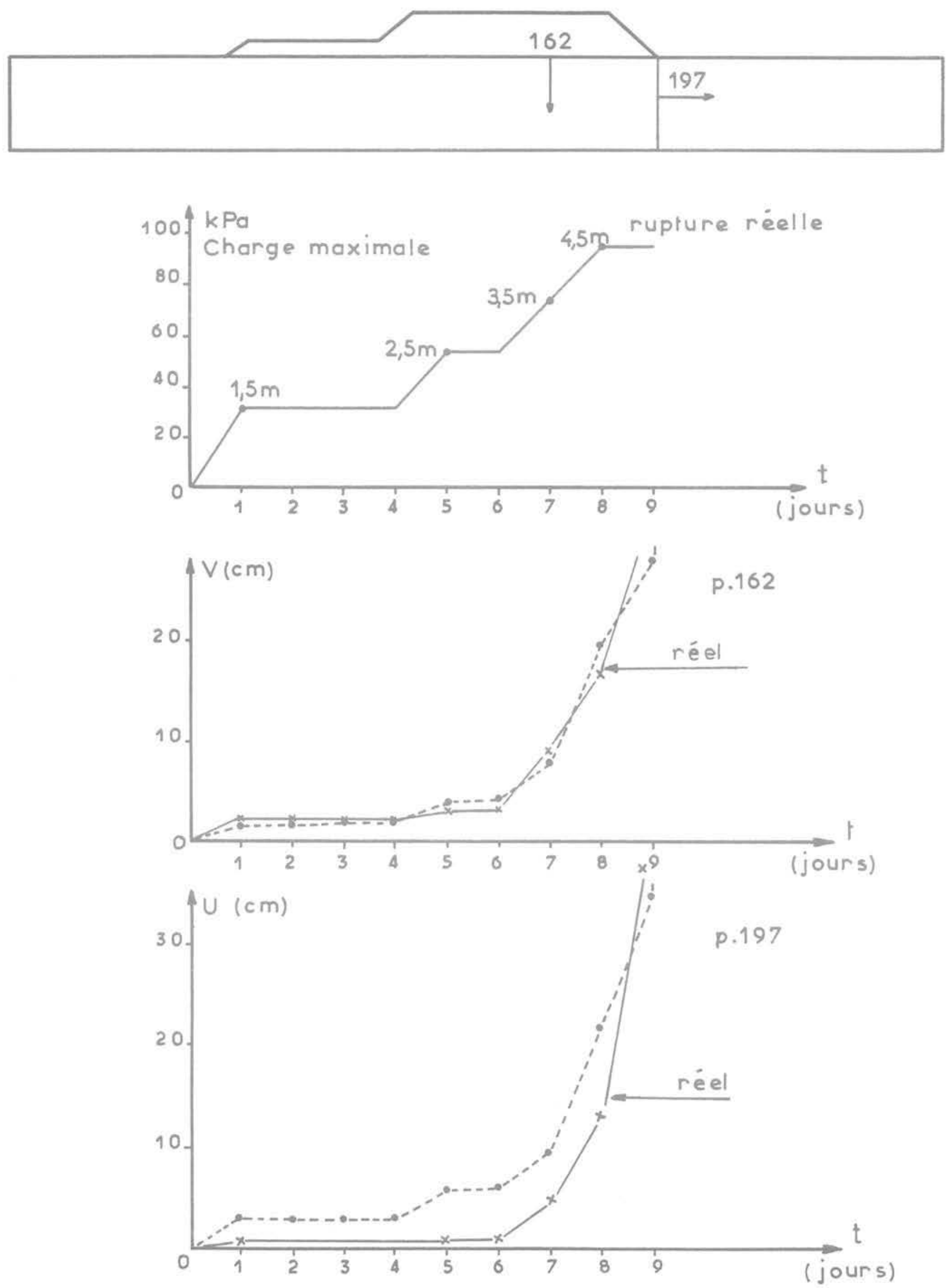

Fig. 7 Remblai A de Cubzac-les-Ponts. Déplacements calculés et observés de deux points du sol de fondation lpoints $n^{\circ} 162$ et 197 du maillage) 

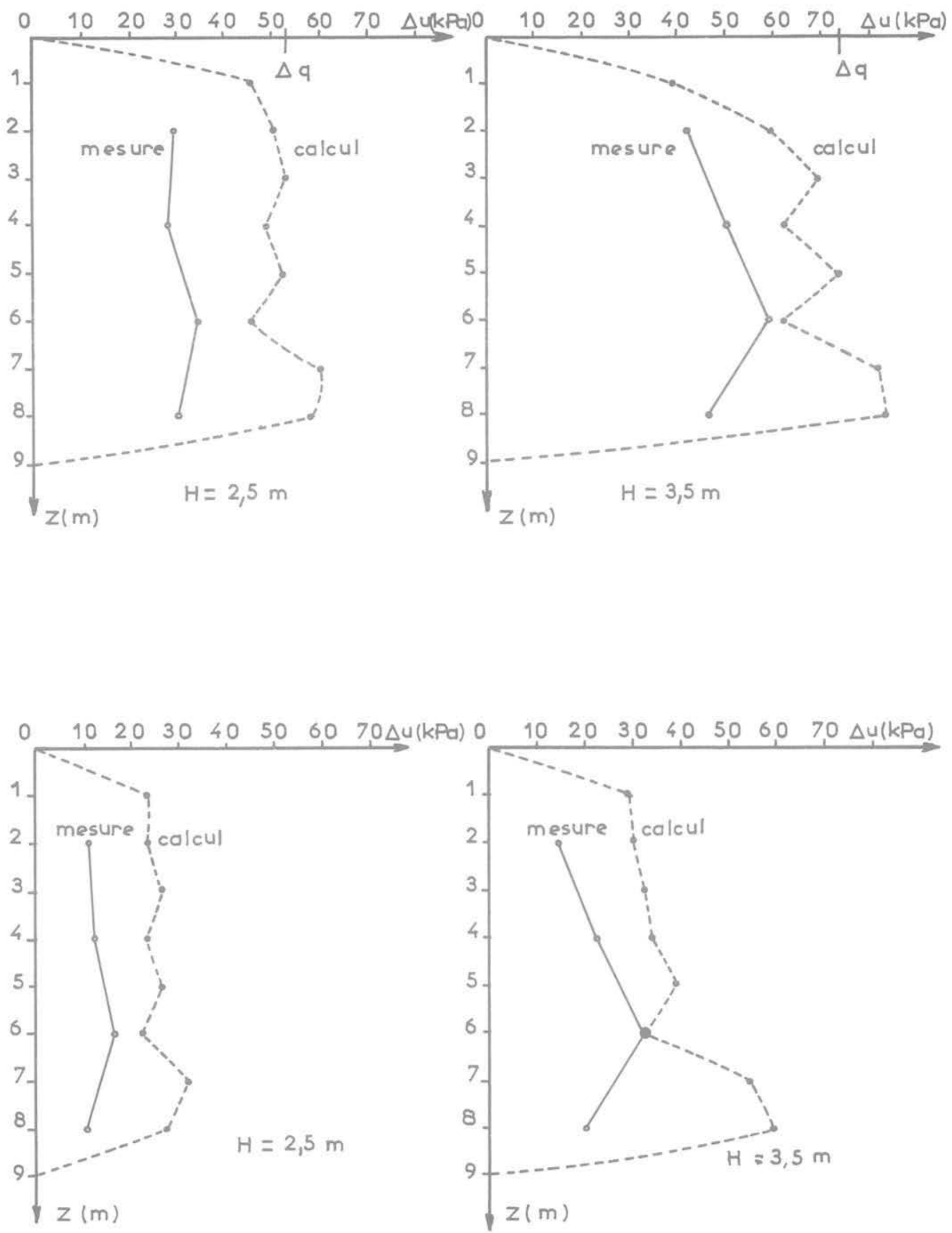

Fig. 8 Remblai A de Cubzac-les-Ponts. Comparaison des surpressions interstitielles calculées et observées sous l'axe du remblai en cours de construction 
S'il se confirme qu'il existe entre $E_{h}, E_{v}, G_{v h}, v_{n h}$ et $v_{v h}$ des relations générales indépendantes du sol testé, la procédure de détermination des paramètres pourra toutefois être simplifiée et à la limite ne plus nécessiter que la mesure du module codométrique.

\section{EXEMPLE D'APPLICATION}

Le modèle Mélanie, tel qu'il a été décrit dans les paragraphes précédents, a été incorporé dans le programme de calcul par élèments finis Rosalie du L.C.P.C. On a pu, ainsi, effectuer des calculs des remblais experimentaux de Cubzac-les-Ponts et comparer les résultats avec les mesures en place. Deux de ces applications, le calcul du remblai $A$ et celui du remblai $B$, ont déjà été publièes (Magnan et al., 1982a, 1982b). Le calcul du remblai A est présenté ci-après dans ses grandes lignes.

Le remblai A, construit en 1974, a été amené jusqu'à la rupture, à une hauteur de $4,5 \mathrm{~m}$. L'argile du site expérimental, qui sert de fondation aux remblais, est a6sez homogène. Il s'agit d'une vase organique, fortement compressible, légèrement surconsolidée et très plastique, dont l'épaisseur varie entre 9 et 9,5 mètres. L'identification géotechnique du sol, réalisée à partir d'essais en place (essais au pénétromètre. au scissomètre, mesure de perméabilité) et d'essais en laboratoire (essais œdométriques, triaxiaux, mesure de $\mathrm{K}_{\mathrm{o}}$ ) a servi de base pour le choix des parametres introduits dans les calculs.

Le maillage utilisé est constitué de 76 éléments quadrilatères répartis en 8 couches suivant I'hétérogénéité du sol. La loi de chargement adoptée pour le calcul suit fidèlement le calendrier de construction et comporte 9 pas de temps de 1 jour chacun. En réalité, la charge finale est atteinte le $8^{\circ}$ jour et la rupture a eu lieu quatre heures après son application. Le comportement calculé du sol dépend des éléments suivants :

- l'état initial, caractérisé par le poids volumique et le coefficient de poussée des terres au repos:

- la perméabilité anisotrope, définie par les deux coefficients de perméabilité $k_{h}$ et $k_{v}$ i

- l'élasticité orthotrope de révolution, dont les paramètres ont été déterminés suivant la méthode exposée au paragraphe précédent:

- l'élastoplasticité avec écrouissage, représentée par l'équation de la surface limite de l'ellipse, pour l'argile de fondation:

- la plasticité parfaite, représentée par l'équation de la droite de Mohr-Coulomb, pour le sable du remblai.

Les résultats du calcul, déplacements horizontaux et verticaux et suppressions interstitielles, ont ensuite été confrontés aux valeurs mesurées en place. Ainsi, la figure 7 montre l'évolution théorique et réelle des déplacements de deux points du massif de fondation. Sur la figure 8 , les valeurs calculées et mesurées des surpressions interstitielles pour deux hauteurs différentes de la plate-forme $(\mathrm{H}=2,5 \mathrm{~m}$ et $\mathrm{H}=3,5 \mathrm{~m})$ sur l'axe de symétrie et sous le pied du talus du remblai sont présentées. Dans les deux cas, on peut constater la bonne concordance des valeurs théoriques et réelles. Le bon fonctionnement du modèle Mélanie a pu être ainsi vérifié dans ce cas d'application. qui présente, outre les difficultés des problèmes classiques de consolidation, les particularités d'une expérience de rupture.

\section{références bibliographiques}

\section{Dang M.-T., Magnan J.-P. (1977)}

Application des modèles élastoplastiques de I'Université de Cambridge au calcul du comportement d'un remblai expérimental sur sols mous. Rapport de Recherche L.P.C. $n^{\circ} 74$, Laboratoire central des Ponts et Chaussées, dêc. 1977.

Leroueil S. (1977)

Quelques considèrations sur le comportement des argiles sensibles, Thèse de doctorat-ès-sciences. Université Laval, Québec.

Magnan J.-P., Belkeziz A. (1982)

Consolidation d'un sol élastoplastique. Revue française de Géotechnique, n 19, mai 1982, pp. 39-49.

Magnan J.-P., Humbert P., Belkeziz A., Mouratidis A. (1982a)

Finite Element Analysis of Soil Consolidation, with Special Reference to the Case of Strain Hardening Elastoplastic Stress-Strain Models, Proc. 4th Intern. Conf. Numerical Methods in Geomechanics, Edmonton, Alberta, Can., may 1982, vol. I. pp. 327336 .

Magnan J.P., Humbert P., Mouratidis A. (1982b)

Finite Element Analysis of Soil Deformations with Time Under an Experimental Embankment, Proc. Intern. Symp. Numerical Models in Geomechanics, Zürich, Balkema, éd., pp. 601-609.

Magnan J.-P., Shahanguian S., Josseaume H. (1982c) Étude en laboratoire des états limites d'une argile molle organique. Revue française de Géotechnique, $n^{\circ} 20$, août 1982, pp. 13-19.

Mouratidis A. (1981)

Modèle élastoplastique anisotrope avec écrouissage pour le calcul des ouvrages sur sols compressibles, Thèse de Docteur-Ingénieur, Université Paris VI.

Piyal M., Magnan J.P. (1984)

Paramètres d'élasticité anisotrope de l'argile molle organique de Cubzac-les-Ponts à l'état surconsolidé. Laboratoire Central des Ponts et Chaussées, Paris, Rapport de Recherche L.P. C.

Roscoe K.H., Burland J.B. (1967)

On the generalized stress-strain behaviour of "wet clay", Symposium on engineering plasticity, Cambridge, University Press, pp. 535-609.

Shahanguian S. (1981)

Détermination expérimentale des courbes d'état limite de l'argile organique de Cubzac-les-Ponts, Rapport de Recherche L.P.C. $n^{\circ} 106$, Laboratoire central des Ponts et Chaussees, Paris.

Tavenas F., Leroueil S. (1977)

Effects of stresses and time on yielding of clays, Comptes-rendus $9^{\circ}$ Congrès Intern. de Mécanique des Sols et des Travaux de Fondations, Tokyo, Vol. 1 , pp. 319-326. 